\title{
SIMULATION OF NON-LINEAR CHARACTERISTICS INFLUENCE DYNAMIC ON VERTICAL RIGID GYRO ROTOR RESONANT OSCILLATIONS
}

\author{
Zharilkassin Iskakov ${ }^{1}$
}

\begin{abstract}
The influence of viscous linear and cubic nonlinear damping of an elastic support on the resonance oscillations of a vertical rigid gyroscopic unbalanced rotor is investigated. Simulation results show that linear and cubic non-linear damping can significantly dampen the main harmonic resonant peak. In non-resonant areas where the speed is higher than the critical speed, the cubic non-linear damping can slightly dampen rotor vibration amplitude in contrast to linear damping. If linear or cubic non-linear damping increase in resonant area significantly kills capacity for absolute motion, then they have little or no influence on the capacity for absolute motion in non-resonant areas. The simulation results can be successfully used to create passive vibration isolators used in rotor machines vibration damping, including gyroscopic ones.
\end{abstract}

UDC Classification: $531.8+62-2$; DOI: http://dx.doi.org/10.12955/cbup.v6.1319

Keyword: gyro rotor, resonant amplitude, linear damping, non-linear damping, mathematical simulation

\section{Introduction}

As is known, rotary machines are widely used in many industry branches and have been studied for a long time. Despite this, there are many outstanding issues, in particular those related to unbalanced mass influence on oscillations and stability and subsequently related to rotary machines resonant oscillations stabilization.

A simplified model of a rotor system with lumped parameters is used generally to study shaft dynamics of a single rotor on bearing supports. It is very important to use support material properties and characteristics for vibration attenuation and damping in order to stabilize the unbalanced rotor and vibration system motion. Supports are by the means of device connected between the rotor and the bearing structure that has different shapes and designs, depending on the specific assumptions. A convenient way is to introduce attenuation for bearings support on visco-resilient elastomeric supports. In parallel with the visco-resilient material simulation development (Gil-Negrete et al., 2009; Richards \& Singh, 1999), which helps to describe material properties complexity, in general use of viscorisilient components in rotor and vibratory systems dynamics (Ravindra \& Mallik, 1994; Peng et al., 2012; Ho et al., 2012) also increased including with non-linear resilient characteristics and damping. Therefore, for example, Ravindra B. and Mallik A. K. (1994) studied the parametric influence of different attenuation types on non-linear vibration isolators performance at harmonic excitation. The efficiency of passive vibration isolators with linear attenuation and cubic non-linear attenuation at resonant and non-resonant oscillation areas was studied in the article by Peng et al. (2012). Here is an excellent overview of linear and non-linear vibration isolation systems study. The influence of material cubic non-linear rigidity on isolator performance was additionally taken into account in the work by Ho et al. (2012). Influences of quadratic non-linear damping on resonant oscillations and stability of vertical unbalanced gyro rotor with resilient support quadratic and cubic non-linear rigidities were studied in the works by Iskakov (2015, October 25; 2017a; 2017b, September 11). Fujiwara et al. (2015, October 25) prepared an experimental single-disk rotor system supported by ball bearings at both ends and compares the vibration with a flexible support containing springs or rubber sheets and with a rigid support base by simulation and experiment.

The influence of bearing resilient support material with linear and cubic non-linear damping on the vertical unbalanced rigid gyro rotor main resonant curve is studied in this work.

\section{Motion equation}

A rotor block diagram, which is illustrated in the Figure 1, is considered. A shaft with $L$ length is installed vertically with lower hinged support and upper resilient support positioned at $l_{0}$ distance from lower hinged support. A disk, which has a mass of $m$, polar inertia moment of $I_{p}$ and roll inertia moment of $I_{T}$ that is identical for all directions is secured at the free shaft end. Shaft rotation speed $\omega$ is so high that rotor can be considered as a gyroscope, whose fixed point is the lower shaft

\footnotetext{
${ }^{1}$ Almaty University of Power Energy and Communications, Institute of Mechanics and Machine Science, iskakov53@mail.ru
} 
support. The position of the disk geometric center $S$ is determined by the coordinates $x, y$ in a fixed coordinate system $O x y z$, coordinates while shaft and entire rotor position in space is determined by $\theta_{x}, \theta_{y}$ angles and by $\varphi=\omega t$ rotation angle. The rotor motion in the direction of the coordinate axis $z$ is neglected. To further denote, the disk mass center $m$ coordinates through $x_{m}$ and $y_{m}$. Also, it is assumed that the linear eccentricity $e$ is on the axis $X$ of the coordinate system $S X Y Z$ rotating together with the rotor. We confine ourselves to small deviations of the rotor axis, hence only taking into account components that are linear with respect to $e, \theta_{x}, \theta_{y}, x_{m}, y_{m}$ and small values in the calculations.

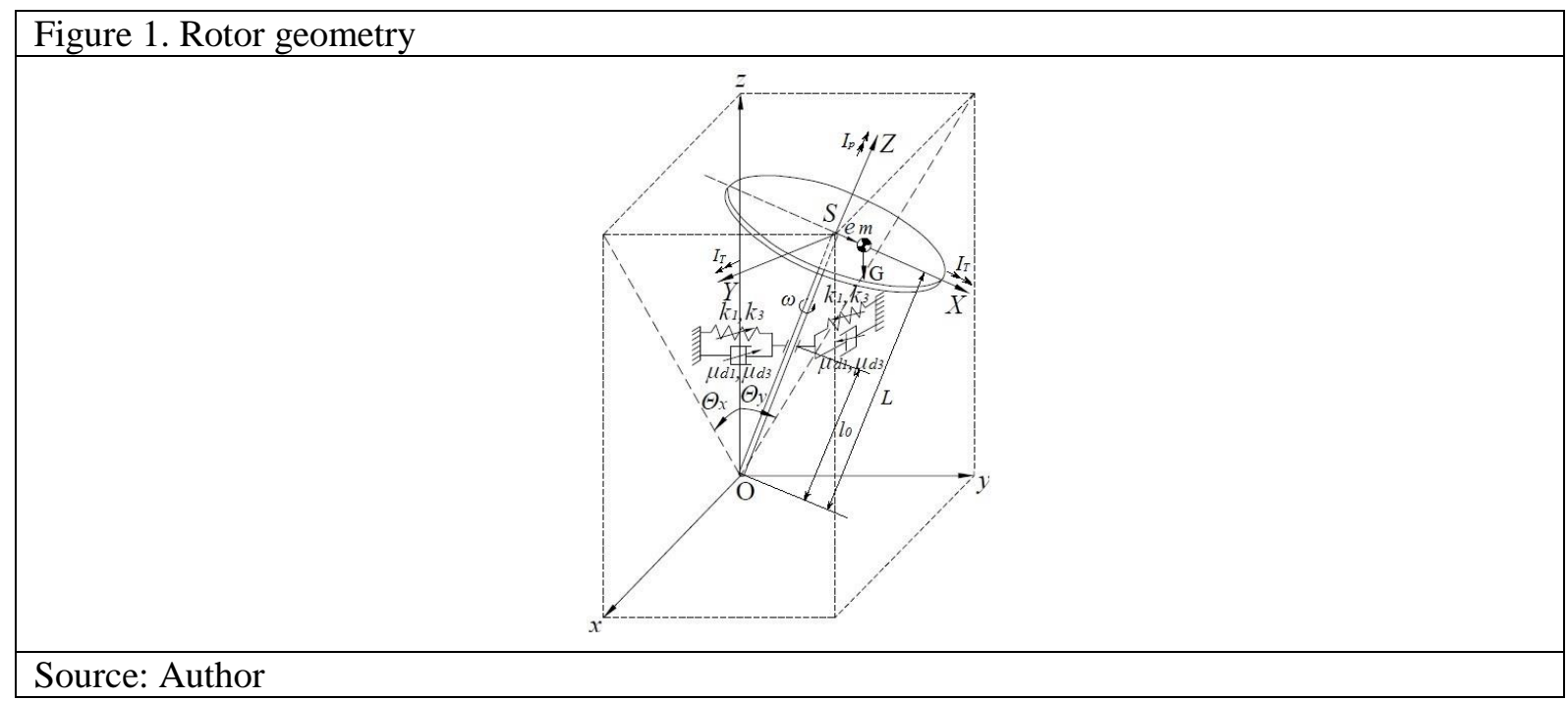

Taking the above into account, angular speed projections on moving system coordinate axes are written in the form of

$$
\omega_{x} \approx-\dot{\theta}_{y}+\omega \theta_{x}, \omega_{y} \approx \dot{\theta}_{x}-\omega \theta_{y}, \omega_{z} \approx \omega+\dot{\theta}_{x} \cdot \theta_{y},
$$

and system kinetic energy

$$
T=\frac{1}{2} m\left(\dot{x}_{m}^{2}+\dot{y}_{m}^{2}\right)+\frac{1}{2}\left(I_{x} \omega_{x}^{2}+I_{y} \omega_{y}^{2}+I_{z} \omega_{z}^{2}\right),
$$

taking into account that $I_{x}=I_{y}=I_{T}, I_{z}=I_{p}$ and (1) we obtain the form of

$$
T=\frac{1}{2} m\left(\dot{x}_{m}^{2}+\dot{y}_{m}^{2}\right)+\frac{1}{2}\left\{I_{T}\left[\left(\dot{\theta}_{x}^{2}+\dot{\theta}_{y}^{2}\right)-2 \omega\left(\theta_{x} \dot{\theta}_{y}+\theta_{y} \dot{\theta}_{x}\right)\right]+I_{p}\left(\omega^{2}+2 \omega \dot{\theta}_{x} \theta_{y}\right)\right\},
$$

where

$$
\begin{aligned}
& x_{m}=x+e \cos \varphi=L \theta_{x}+e \cos \omega t, \\
& y_{m}=y+e \sin \varphi=L \theta_{y}+e \sin \omega t .
\end{aligned}
$$

System potential energy is of the form of

$$
V=\frac{1}{2} k_{1} l_{0}^{2}\left(\theta_{x}^{2}+\theta_{y}^{2}\right)+\frac{1}{4} k_{3} l_{0}^{4}\left(\theta_{x}^{4}+\theta_{y}^{4}\right),
$$

where $k_{1}$ is the support rigidity coefficient, $k_{3}$ is the coefficient in elastic force non-linear term.

External forces moments are of the form of

$$
M_{x}=\left(L \theta_{x}+e \cos \omega t\right) G, M_{y}=\left(L \theta_{y}+e \sin \omega t\right) G,
$$

where $G=m g$ is disk mass, $g$ - gravitational acceleration.

Dissipation forces are considered and defined in the form of

$$
\Phi=\frac{1}{2} \mu_{d 1}\left(\dot{\theta}_{x}^{2}+\dot{\theta}_{y}^{2}\right)+\frac{1}{4} \mu_{d 3}\left(\dot{\theta}_{x}^{4}+\dot{\theta}_{y}^{4}\right),
$$


where $\mu_{d 1}$ is the linear viscous damping coefficient; $\mu_{d 3}$ is the non-linear cubic viscous damping coefficient.

Lagrange's equations of the second kind (Yablonsky, 2007) for rotor systems are set out in the form of

$$
\frac{d}{d t}\left(\frac{\partial T}{\partial \dot{q}_{i}}\right)-\frac{\partial T}{\partial q_{i}}+\frac{\partial V}{\partial q_{i}}=-\frac{\partial \Phi}{\partial \dot{q}_{i}}+Q_{i} .
$$

Here $q_{i}: \theta_{x}, \theta_{y}$ are generalized coordinates, $Q_{i}: M_{x}, M_{y}$ are generalized forces.

By substituting the expression (2)-(6) to (7), we obtained the motion equations of the rotor

$$
\begin{aligned}
& \left(I_{T}+m L^{2}\right) \ddot{\theta}_{x}+I_{P} \omega \dot{\theta}_{y}+\mu_{d 1} \dot{\theta}_{x}+\mu_{d 3} \dot{\theta}_{x}^{3}+\left(k_{1} l_{0}^{2}-G L\right) \theta_{x}+k_{3} l_{0}^{4} \theta_{x}^{3}=\left(m e \omega^{2} L+G e\right) \cos \omega t, \\
& \left(I_{T}+m L^{2}\right) \ddot{\theta}_{y}-I_{P} \omega \dot{\theta}_{x}+\mu_{d 1} \dot{\theta}_{y}+\mu_{d 3} \dot{\theta}_{y}^{3}+\left(k_{1} l_{0}^{2}-G L\right) \theta_{y}+k_{3} l_{0}^{4} \theta_{y}^{3}=\left(m e \omega^{2} L+G e\right) \sin \omega t,
\end{aligned}
$$

where

$$
m e \omega^{2} L+G e=M_{f}
$$

- amplitude of the resultant moment of external forces.

Work following dimensionless parameters in

$$
\begin{aligned}
& \varepsilon=e / L ; l=l_{0} / L ; \bar{t}=t \omega_{0} ; \Omega=\omega / \omega_{0} ; \bar{I}_{p}=I_{p} /\left(m L^{2}\right) ; \bar{I}_{T}=I_{T} /\left(m L^{2}\right) ; \bar{K}_{1}=k_{1} /\left(m \omega_{0}^{2}\right) ; \\
& \bar{K}_{3}=k_{3} L^{2} /\left(m \omega_{0}^{2}\right) ; P=G /\left(m L \omega_{0}^{2}\right) ; \bar{\mu}_{1}=\mu_{d 1} /\left(m L^{2} \omega_{0}\right) ; \bar{\mu}_{3}=\mu_{d 3} \omega_{0} /\left(m L^{2}\right),
\end{aligned}
$$

where

$$
\omega_{0}=\sqrt{\frac{k_{1} l_{0}^{2}-G L}{m L^{2}-\left(I_{p}-I_{T}\right)}}
$$

is non-damping linear system critical speed, using designation for the dimensionless amplitude expression of the resultant moment of external forces

$$
\bar{M}=\varepsilon \Omega^{2}+\varepsilon P
$$

it is possible to make motion equations (8) compact and dimensionless

$$
\begin{aligned}
& \left(1+\bar{I}_{T}\right) \theta_{x}^{\prime \prime}+\bar{I}_{p} \Omega \theta_{y}^{\prime}+\bar{\mu}_{1} \theta_{x}^{\prime}+\bar{\mu}_{3} \theta_{x}^{\prime 3}+\left(\bar{K}_{1} l^{2}-P\right) \theta_{x}+\bar{K}_{3} l^{4} \theta_{x}^{3}=\bar{M} \cos \Omega \bar{t}, \\
& \left(1+\bar{I}_{T}\right) \theta_{y}^{\prime \prime}-\bar{I}_{p} \Omega \theta_{x}^{\prime}+\bar{\mu}_{1} \theta_{y}^{\prime}+\bar{\mu}_{3} \theta_{y}^{\prime 3}+\left(\bar{K}_{1} l^{2}-P\right) \theta_{y}+\bar{K}_{3} l^{4} \theta_{y}^{3}=\bar{M} \sin \Omega \bar{t} .
\end{aligned}
$$

Here the dashes denote the derivatives with respect to the dimensionless time $\bar{t}$.

Thus, it occurs that the state of the concerned rotor steady motion is described by differential equation system (13) and (14) of Duffing type. Usually, a method of expansion of solutions (13) and (14) to a Fourier series with uncertain coefficients is used to determine the periodic solution with a period equal to external influence period. The coefficients can be obtained with a harmonic balance method (Hayashi, 1964; Szemplinska-Stupnicka, 1968; Kydyrbekuly, 2006) while taking into account the finite and usually small amount of terms.

\section{Oscillations AFC and PFC}

A gyro rotor with rigid non-linear resilient characteristic and cubic non-linear damping is studied for resonance at the main frequency.

Solution approximations of equations (13) and (14) in the case when main resonance is a simple harmonic with oscillations frequency equal to disturbing moment frequency complies with

$$
\begin{aligned}
& \theta_{x}=A \cos (\Omega \overline{\mathrm{t}}-\alpha), \\
& \theta_{y}=A \sin (\Omega \overline{\mathrm{t}}-\alpha) .
\end{aligned}
$$

We obtained amplitude- and phase-frequency dependencies of the fundamental harmonic after applying harmonic balance method (Hayashi, 1964; Szemplinska-Stupnicka, 1968; Kydyrbekuly, 2006)

$$
\left\{\left[(1-H) \Omega^{2}-\left(\bar{K}_{1} l^{2}-P\right)-0,75 \bar{K}_{3} l^{4} A^{2}\right]^{2}+\left[\bar{\mu}_{1}+0,75 \bar{\mu}_{3} A^{2} \Omega^{2}\right]^{2} \Omega^{2}\right\} A^{2}=\bar{M}^{2},
$$




$$
\tan \alpha=\frac{\left(\bar{\mu}_{1}+0,75 \bar{\mu}_{3} A^{2} \Omega^{2}\right) \Omega}{-(1-H) \Omega^{2}+\left(\bar{K}_{1} l^{2}-P\right)+0,75 \bar{K}_{3} l^{4} A^{2}},
$$

where $H=\bar{I}_{p}-\bar{I}_{T}$ is the conditional disk thickness.

Results for rotor linear model (Iskakov \& Kalybayeva, 2010, September 14) were obtained from expressions (17) and (18) when there are no non-linear terms in equations (13) and (14).

We obtained expressions for amplitude- and phase-frequency characteristics by

$$
1=\left(\bar{K}_{1} l^{2}-P\right) /(1-H) ; K_{3}=\bar{K}_{3} /(1-H) ; \mu_{1}=\bar{\mu}_{1} /(1-H), \mu_{3}=\bar{\mu}_{3} /(1-H) ; M=\bar{M} /(1-H)
$$

working additional values notations in

$$
\begin{gathered}
\left(\omega_{*}^{2}-\Omega^{2}\right)^{2}+\left(\mu_{1} \Omega+0,75 \mu_{3} \Omega^{3} A^{2}\right)^{2}=M^{2} / A^{2}, \\
\tan \alpha=\frac{\left(\mu_{1}+0,75 \mu_{3} A^{2} \Omega^{2}\right) \Omega}{1-\Omega^{2}+0,75 K_{3} l^{4} A^{2}},
\end{gathered}
$$

where following notations are worked in

$$
\omega_{*}^{2}=1+0,75 K_{3} l^{4} A^{2} .
$$

Amplitude-frequency supporting curves usually describe the relationship between amplitude and the frequency of system free oscillations without damping (Panovko, 1971). We obtained a supporting curve equation for oscillations at the main resonance frequency assuming that expression $M$ is caused by an external moment, and the damping coefficients $\boldsymbol{\mu}_{1}$ and $\boldsymbol{\mu}_{3} \mathrm{n}$ the equations (20) are equal to zero

$$
A=\frac{1}{\sqrt{0,75 K_{3}} l^{2}} \sqrt{\Omega^{2}-1} \text {. }
$$

Here

$$
\Omega \geq 1 \text {. }
$$

It is obvious from formula (23) that the supporting curve is a parabola that is symmetrically relative to the $\Omega$ axis, and greater values of $K_{3}$ result in greater inclination to the right of the supporting curve.

\section{Damping influence on rotor dynamics}

In case when $H=0,99$ and moment $P$ of gravitational force is neglected the amplitude of the centrifugal couple is $M=\Omega^{2}$. We obtained graphs in Figure 2 by solving equation (20) relatively to $A$ depending on $\Omega$ and $\mu_{1}$ at constant value of $\mu_{3}$, and depending on $\Omega$ and $\mu_{3}$ at constant value of $\mu_{1}$ with $K_{3}=0$, it is obvious that an increase of linear damping coefficient $\boldsymbol{\mu}_{1}$ can considerably dampen amplitude $A$ of the fundamental harmonic in the resonant area, where $\Omega \approx 1$, however influence of $\mu_{1}$ on amplitude $A$ in the non-resonant areas, where $\Omega \succ 1$ or $\Omega \prec 1$ is very weak and negligible (Figure 2 a). One can see that an increase of cubic non-linear damping coefficient $\mu_{3}$ can considerably dampen amplitude $A$ of the fundamental harmonic in the resonant area where $\Omega \approx 1$, but $\mu_{3}$ has little or no influence on amplitude $A$ in non-resonant area, where $\Omega \prec 1$. However, in the non-resonant area where $\Omega \succ 1$, one can see that an increase of $\boldsymbol{\mu}_{3}$ can slightly dampen amplitude $A$ of oscillation (Figure $2 \mathrm{~b}$ ).

We obtained an expression to determine of the transmissibility force amplitude depending on $\mu_{1}$ with the constant value of $\mu_{3}$, and also on $\mu_{3}$ with the constant value of $\mu_{1}$ by solving the equations of the transmissibility force in the projections with harmonic balance method

$$
A_{\tau}^{2}=\left(\mu_{1} A \Omega+0,75 \mu_{3} A^{3} \Omega^{3}\right)^{2}+\left(A+0,75 K_{3} A^{3}\right)^{2} .
$$

Graphs of these dependences are illustrated in Figure 3. It is obvious from these graphs that in the nonresonant area $\Omega \prec 1$ the linear damping $\boldsymbol{\mu}_{1}$ and cubic non-linear damping $\mu_{3}$ have little or no influence on isolator capability for absolute motion. In the resonance area where $\Omega \approx 1$, an increase either in the linear damping $\mu_{1}$, or in cubic non-linear damping $\mu_{3}$ can considerably dampen the capability for absolute motion. In the non-resonant area $\Omega \succ \sqrt{2}$ an increase of $\mu_{3}$ can increase $A_{\tau}$ a little, but linear damping $\boldsymbol{\mu}_{\mathbf{1}}$ has little or no influence on $A_{\tau}$. 
Thus, it is possible to create such rigid support, which would render simultaneously attenuating and damping influence on the resonant amplitude and would give the possibility for the rotor to pass its critical speed without serious consequences by trying the rigidity coefficient $k$, linear damping coefficient $\mu_{d 1}$ and non-linear cubic damping coefficient $\mu_{d 3}$ values of the support. Attenuating and damping properties of the support are resilient supports action principle of the centrifuge operating on gyro rotor base, for which the Republic of Kazakhstan invention patent was obtained (Iskakov \& Kunelbayev, 2018).
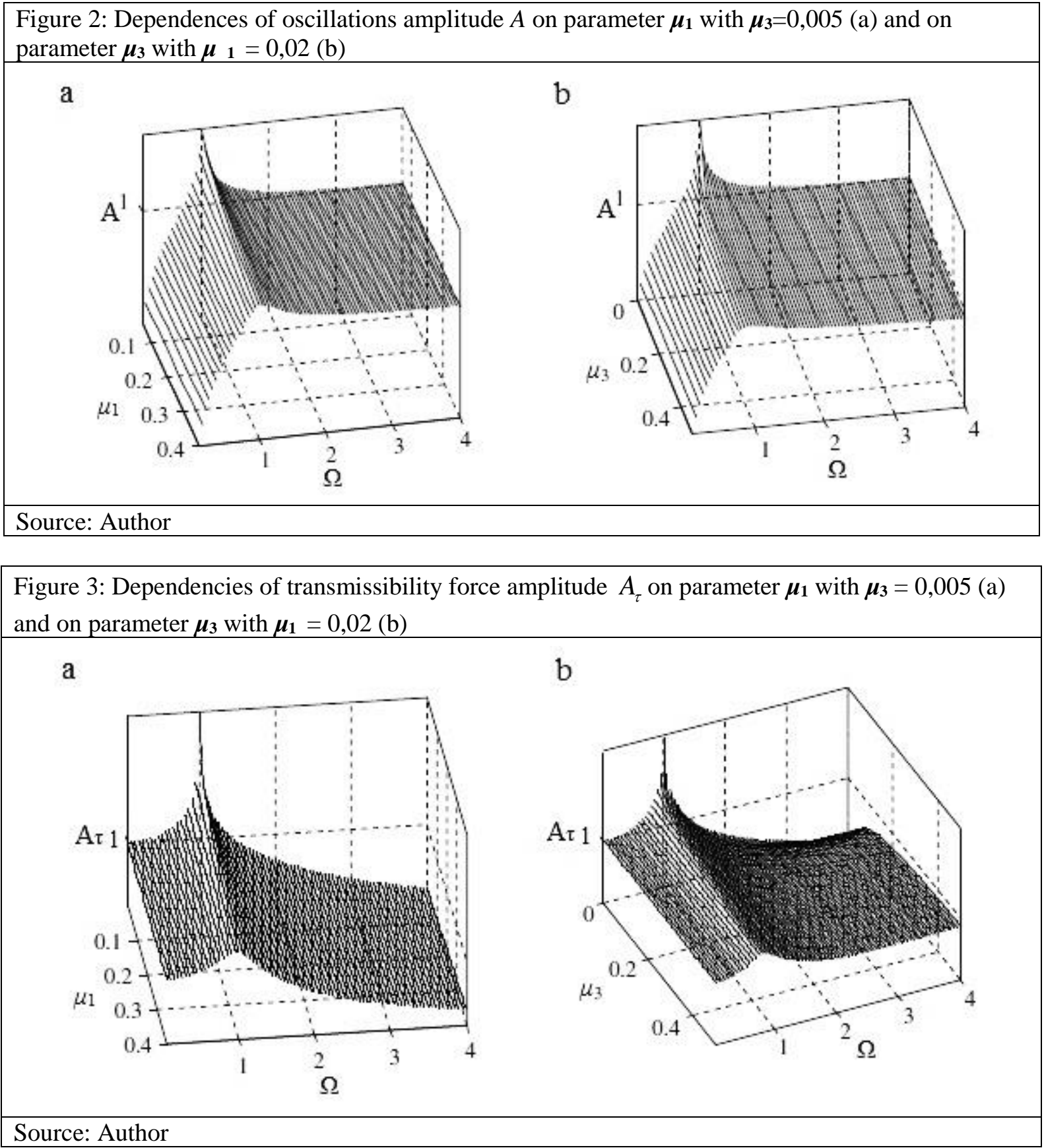

The invention is illustrated in drawings in Figure 4. In Figure 4, the drive electric motor 4 with powertakeoff shaft 5 is secured with a cylindrical ring 1 and brackets 2 on the platform 3 . The electric motor shank is connected to the power-takeoff shaft with the adapter coupling 6 . The drive electric motor with power-takeoff shaft is secured to the frame with bottom hinge 7 and upper spring 8 and rubber 9 resilient supports. Cylindrical vessel 10, held on the shaft 5 by the lock nut 11 is installed on the upper conical part of the shaft 5. Cylindrical vessel has inner casing 12 and it is covered by the transparent cover 13. Motion sensors 14 connected with the control device by wires 15,16 and 17 are installed on 
the bracket 2 to measure processional motion. Magnetoelectric revolution sensors 18 , connected by wires 19, 20 with the recording device in the control device are used to measure shaft 5 speed. Rotor speed in operational mode is set up by the power source voltage regulator. Power to electric motor is supplied by wires 21 and 22. Shaft precession amplitude for operating speed and at full liquid prime is set up by tying the upper resilient support spring 8 with nut 23 depending on technological process and production quality requirements. In addition, a lower resilient support 9 rubber cap is selected so that the vibration isolation coefficient of the specially manufactured rubber or elastic material was less than one with the purpose of safe passage of critical speed in the case when the operating speed was determined to be beyond critical speed.

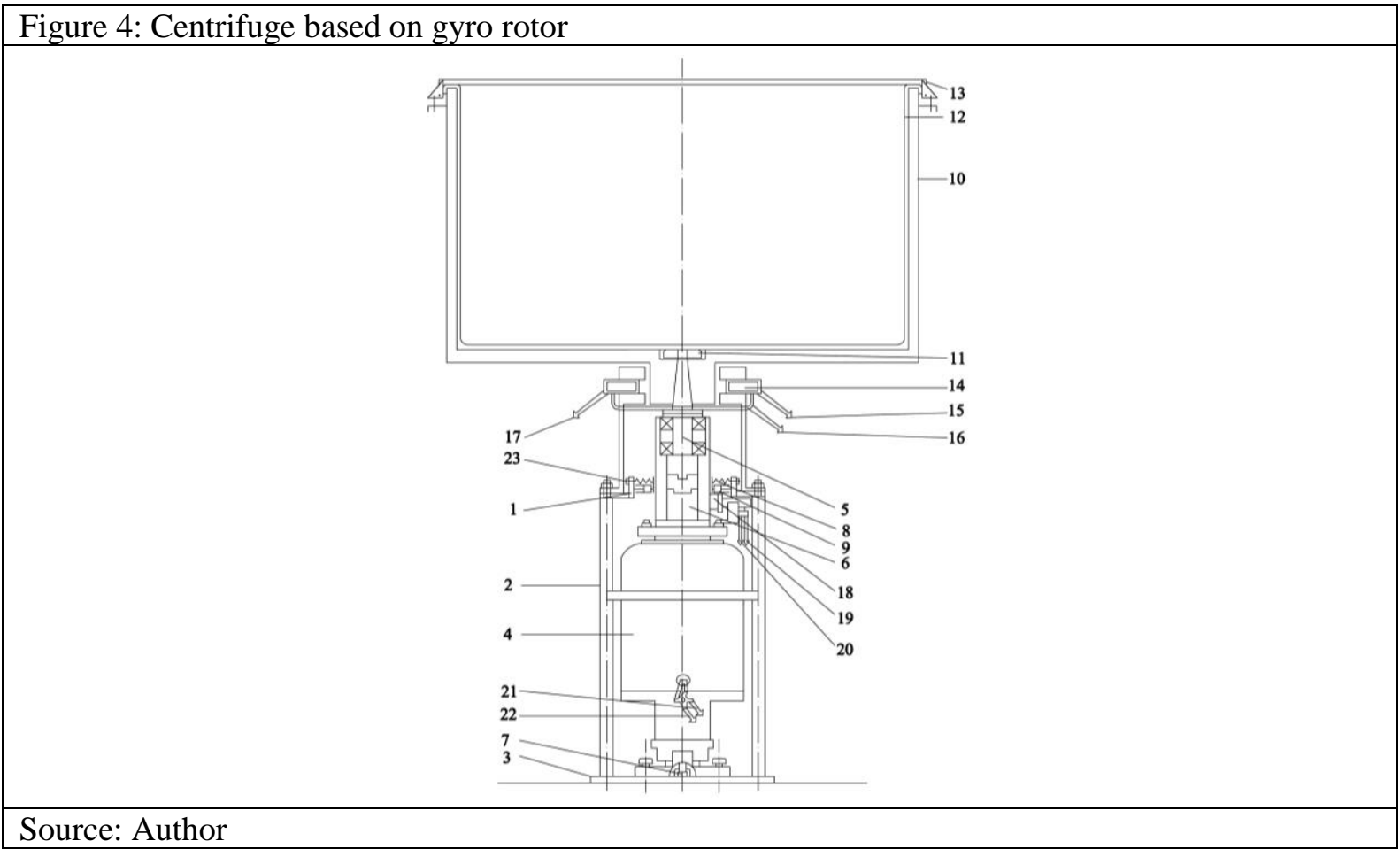

\section{Conclusion}

The influence of bearing resilient support material with linear viscous damping and with the cubic non-linear viscous damping on vertical rigid gyro rotor resonant oscillations was studied in the work. Rotor motion equations were composed on the basis of Lagrange's equations of the second type, which were reduced to the dimensionless form. Rotor motion equations were solved by a harmonic balance method and amplitude-frequency and phase-frequency characteristics of the main harmonic expressions were obtained. The amplitude-frequency characteristic studies depending on resilient supports linear viscous damping and non-linear viscous cubic damping coefficients show that linear and non-linear cubic damping significantly dampen resonant amplitude of the main harmonic. In nonresonant areas, influence of linear damping on vibration is weak and negligible and non-linear cubic damping in areas where the speed is higher than the critical speed can slightly dampen rotor oscillations amplitude. The results of the studies were used to simulate the operation principle of centrifuge gyro rotor resilient support, for which an invention patent was obtained.

\section{References}

Fujiwara, H., Nakaura, H., \& Watanabei K. (2015). The vibration behavior of flexibly fixed rotating machines, Proceedings of the 14th IFToMM World Congress. Taipei, Taiwan.

Gil-Negrete, N., Vinolas, J., \& Kari L. (2009). A non-linear rubber material model combining fractional order viscoelasticity and amplitude dependent effects, Journal of Applied Mechanics, 76(1), 9 -11.

Hayashi, C. (1964). Non-linear Oscillations in Physical Systems (Chapters 1, 3 - 6). McGraw - Hill.

Ho, C., Lang Z., \& Billings, S. A. (2012). The benefits of non-linear cubic viscous damping on the force transmissibility of a Duffing-type vibration isolator. Proceedings of UKACC International Conference on Control, 479-484. Cardiff, UK.

Iskakov Zh. \& Kalybayeva A. (2010, September 14). Kolebaniya i ustoychivost' vertikal'nogo giroskopicheskogo rotora s perekosom diska i disbalansom massy [Oscillations and stability of vertical gyro rotor with disk tilt and mass disbalance]. 
Trudy I Mezhdunarodnogo simposiuma: "Fundamental'nue i prikladnye problemy" [Proc. of International Symposium "Fundamental and Applied Science Problems"] Moscow pp. 50 - 57. RAS.

Iskakov Zh. \& Kunelbayev M. (2018). Centrifuga na baze gyroskopicheskogo rotora [Centrifuge based on gyro rotor]. Invention patent of the Republic of Kazakhstan (19) KZ B (11) 32666 19.02.2018, bulletin № 7.

Iskakov, Zh. (2015, October 25). Resonant Oscillations of a Vertical Unbalanced Gyroscopic Rotor with Non-linear. Characteristics. Proceedings of the 14th IFToMM World Congress. Taipei, Taiwan. DOI Number: 10.6567/IFToMM.14TH.WC.OS14.001.

Iskakov, Zh. (2017a). Dynamics of a Vertical Unbalanced Gyroscopic Rotor with Non-linear Characteristics. New advances in Mechanisms, Mechanical Transmissions and Robotics, Mechanisms and Machine Science, 46, 107-114. Springer International Publishing AG. DOI 10.1007/978-319-45450-4_11.

Iskakov, Zh. (2017b, September 11). Rezonansnyye kolebaniya neuravnoveshennogo vertikal'nogo giroskopicheskogo rotora s nelineynymi kharakteristikami [Resonant oscillations of non-balanced vertical gyro rotor with non-linear characteristics]. Proceedings of the International Symposium of Mechanism and Machine, 240-246. Science, Baku.

Kyderbekuly A.B. (2006). Kolebaniya i ustoychivost' rotornykh sistem i ploskikh mekhanizmov s nelineyno- uprugimi kharakteristikami [Oscillations and stability of rotor systems and plane mechanisms with non-linear rigid characteristics] (Doctor of Engineering Science Dissertation.:01.02.06). Almaty.

Panovko Ya. G. (1971). Vvedeniye v teoriyu mekhanicheskikh kolebaniy [Introduction to mechanical oscillations theory]. Moscow, Nauka.

Peng, Z. K., Mengand Lang, Z. Q., Zhang, W. M., \& Chu, F. L. (2012). Study of the effects of cubic non-linear damping on vibration isolations using Harmonic Balance Method. International Journal of Non-Linear Mechanics, 47(10), 1065-1166.

Ravindra, B., \& Mallik, A. K. (1994). Performance of non-linear vibration isolators under harmonic excitation. Journal of Sound and Vibration, 170, 325-337.

Richards, C. M., \& Singh, R. (1999). Experimental characterization of non-linear rubber isolators in a multi-degree-offreedom system configuration. The journal of the Acoustical Society of America, 106. 21 - 78.

Szemplinska-Stupnicka, W. (1968). Higher harmonic oscillations in heteronymous non-linear systems with one degree of freedom. International Journal of Non-Linear Mechanics, 3(1), 17-30.

Yablonskiy A.A. (2007). Kurs teorii kolebaniy [Oscillation theory course]. Study guide. BHV - St. Petersburg. 